Clinical paper

\title{
Cerebral saturation in cardiac arrest patients measured with near-infrared technology during pre-hospital advanced life support. Results from Copernicus I cohort study
}

\author{
Cornelia Genbrugge $^{\mathrm{a}, \mathrm{b}, \mathrm{c}, *}$, Cathy De Deyne ${ }^{\mathrm{a}, \mathrm{b}, \mathrm{c}}$, Ward Eertmans ${ }^{\mathrm{a}, \mathrm{b}, \mathrm{c}}$, Kurt Anseeuw ${ }^{\mathrm{d}}$, Dirk Voet , $^{\mathrm{e}}$, \\ Ilse Mertens ${ }^{\mathrm{f}}$, Marc Sabbe ${ }^{\mathrm{g}}$, Jan Stroobants ${ }^{\mathrm{h}}$, Liesbeth Bruckers ${ }^{\mathrm{i}}$, Dieter Mesotten ${ }^{\mathrm{b}}$, Frank Jans ${ }^{\mathrm{a}, \mathrm{b}, \mathrm{c}}$, \\ Willem Boer ${ }^{\mathrm{b}, \mathrm{c}}$, Jo Dens ${ }^{\mathrm{a}, \mathrm{c}, \mathrm{j}}$

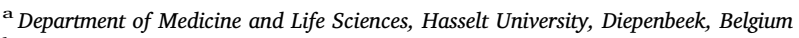 \\ ${ }^{\mathrm{b}}$ Department of Anaesthesiology, Intensive Care, Emergency Medicine and Pain Therapy, Ziekenhuis Oost-Limburg, Genk, Belgium \\ ${ }^{\mathrm{C}}$ Schiepse Bos 6, 3600, Genk, Belgium \\ d Emergency Department, Ziekenhuis Netwerk Antwerpen, Campus Stuivenberg, Lange Beeldekensstraat 267, 2060, Antwerpen, Belgium \\ emergency Department, Gasthuiszusters Antwerpen, Campus Sint-Vincentius, Sint-Vincentiusstraat 20, 2018, Antwerpen, Belgium \\ ${ }^{\mathrm{f}}$ Emergency Department, Algemeen Ziekenhuis Turnhout, Campus Sint-Elisabeth, Rubensstraat 166, 2300, Turnhout, Belgium \\ ${ }^{g}$ Emergency Department, University Hospitals Leuven, Leuven, Belgium \\ ${ }^{\mathbf{h}}$ Emergency Department, Ziekenhuis Netwerk Antwerpen, Campus Middelheim, Lindendreef 1, 2020, Antwerpen, Belgium \\ ${ }^{\mathrm{i}}$ I-Biostat (CenStat), Hasselt University, Agoralaan gebouw D, 3590, Diepenbeek, Belgium \\ ${ }^{\mathbf{j}}$ Department of Cardiology, Ziekenhuis Oost-Limburg, Genk, Belgium
}

\section{A R T I C L E I N F O}

\section{Keywords:}

Cerebral saturation

Cardiac arrest

Out-of hospital cardiac arrest

Pre-hospital

Cerebral oximetry

Advanced life support

\begin{abstract}
A B S T R A C T
Aim: To date, monitoring options during pre-hospital advanced life support (ALS) are limited. Regional cerebral saturation $\left(\mathrm{rSO}_{2}\right)$ may provide more information concerning the brain during ALS. We hypothesized that an increase in $\mathrm{rSO}_{2}$ during ALS in out-of hospital cardiac arrest (OHCA) patients is associated with return of spontaneous circulation (ROSC).

Methods: A prospective, non-randomized multicenter study was conducted in the pre-hospital setting of six hospitals in Belgium. Cerebral saturation was measured during pre-hospital ALS by a medical emergency team in OHCA patients. Cerebral saturation was continuously measured until ALS efforts were terminated or until the patient with sustained ROSC ( $>20 \mathrm{~min}$ ) arrived at the emergency department. To take the longitudinal nature of the data into account, a linear mixed model was used. The correlation between the repeated measures of a patient was handled by means of a random intercept and a random slope. Our primary analysis tested the association of $\mathrm{rSO}_{2}$ with ROSC.

Results: Of the 329 patients 110 (33\%) achieved ROSC. First measured $\mathrm{rSO}_{2}$ was 30\% \pm 18 in the ROSC group and $24 \% \pm 15$ in the no-ROSC group ( $\mathrm{p}=.004$; mean $\pm \mathrm{SD}$ ). Higher mean $\mathrm{rSO}_{2}$ values were observed in the ROSC group compared to the no-ROSC group $(41 \% \pm 13$ versus $33 \% \pm 13$ respectively; $p<0.001)$. The median increase in $\mathrm{rSO}_{2}$, measured from start until two minutes before ROSC, was higher in the ROSC group (ROSC group 17\% (IQR 6-29)) than in the no-ROSC group (8\% (IQR 2-13); p < 0.001). An increase in $\mathrm{rSO}_{2}$ above 15\% was associated with ROSC (OR 4.5; 95\%CI 2.747-7.415; p < 0.001).

Conclusion: Regional cerebral saturation measurements can be used during pre-hospital ALS as an additional marker to predict ROSC. An increase of at least $15 \%$ in $\mathrm{rSO}_{2}$ during ALS is associated with a higher probability of ROSC.
\end{abstract}

\footnotetext{
A Spanish translated version of the abstract of this article appears as Appendix in the final online version at https://doi.org/10.1016/j.resuscitation.2018.03.031.

* Corresponding author at: Cornelia Genbrugge Maria-theresiastraat 34/3, 3000, Leuven, Belgium.

E-mail addresses: cornelia.genbrugge@uhasselt.be (C. Genbrugge), cathy.dedeyne@zol.be (C. De Deyne), ward.eertmans@uhasselt.be (W. Eertmans),

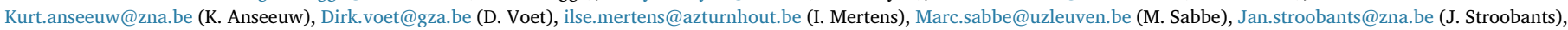
Liesbeth.bruckers@uhasselt.be (L. Bruckers), Dieter.mesotten@zol.be (D. Mesotten), frank.jans@zol.be (F. Jans), willem.boer@zol.be (W. Boer), jo.dens@zol.be (J. Dens).
} 


\section{Introduction}

The goal of advanced life support (ALS) is to restore coronary perfusion and regain pulses. In this manner, it will preserve the pre-arrest neurologic state by maintaining sufficient cerebral blood flow (CBF) and oxygenation. However, post-anoxic brain injury remains the primary cause of death in initially resuscitated OHCA patients $[1,2]$.

Current monitoring options during ALS are limited to rhythm assessments, pulse checks and measurements of end-tidal carbon dioxide $\left(\mathrm{ETCO}_{2}\right)$ [3]. These monitoring modalities have major shortcomings. Rhythm assessments and pulse checks require interruptions of chest compressions, and most importantly, none of the available monitoring options provide direct information about oxygen supply to vital organs, especially the brain $[1,2]$. None of the current methods is perfect. Moreover, the absence of specific and reliable information on the brain during ALS leaves the physician unaware of the efficiency of ALS on the brain.

Measurement of cerebral saturation $\left(\mathrm{rSO}_{2}\right)$ is a non-invasive technique that uses near infrared spectroscopy (NIRS) to measure the oxygen saturation in the superficial parts of the frontal brain lobes. Although this technique is primarily used in the per-operative setting and neonatal intensive care, it has recently been introduced in the prehospital cardiac arrest (CA) setting [4-6]. Moreover, its use has been previously studied in in-hospital cardiac arrest patients as well as in OHCA patients after arrival at the emergency department [7-10]. Cerebral saturation may provide information concerning the brain during ALS as it is non-invasive, real time, continuous and independent of a pulsatile signal and in this manner addresses a clinically important problem. It could be of an added value when used in conjunction with current monitoring options.

To date, no multicenter study has been performed in an OHCA setting, which investigated the use of $\mathrm{rSO}_{2}$ monitoring during ALS to predict return of spontaneous circulation (ROSC). In the Copernicus multicenter cohort trial (NCT 01806844), we hypothesized that an increase in $\mathrm{rSO}_{2}$ during ALS in OHCA patients is associated with ROSC.

\section{Material and methods}

This prospective, non-randomized multicenter study was conducted in the pre-hospital setting of six hospitals in Belgium (Ziekenhuis OostLimburg, Genk, University Hospitals Leuven, Ziekenhuis Netwerk Antwerpen Middelheim, Ziekenhuis Netwerk Antwerpen Stuivenberg, Algemeen Ziekenhuis Turnhout and Gasthuiszusters Antwerpen StVincentius). In Belgium, every hospital with a pre-hospital medical emergency team is responsible for all pre-hospital incidents in a certain perimeter. The study was initiated first of December 2011 and ended November 15th 2015. The study protocol was approved by the ethics committees of each participating institution (13/044U) and was registered at ClinicalTrials.gov (NCT 01806844). The requirement for informed consent was waived.

\section{Study design and protocol}

Cerebral saturation was measured continuously in OHCA patients during pre-hospital ALS by a medical emergency team. The pre-hospital medical emergency team uniformly consisted of a nurse and a physician. In addition, there was support from an ambulance crew, made up of either two paramedics or one paramedic and one emergency nurse. Paramedics performed only BLS and were not allowed to administer any drugs or intubate. The pre-hospital medical emergency team was equipped with a portable cerebral oximeter Equanox $^{\mathrm{TM}} 7600$ or SenSmart ${ }^{\mathrm{TM}}$ Model X-100, Nonin Medical Inc, Plymouth, MN, USA). The accuracy and reliability of the used cerebral oximeters have been validated against a mix of $70 \%$ jugular bulb saturation and $30 \%$ arterial saturation [11]. The emergency physician applies only one $\mathrm{rSO}_{2}$ sensor to minimize time delay. This single sensor is placed on the right side of the forehead of the patient, without cleaning the skin. All patients are ventilated with $100 \%$ oxygen. ALS is performed according to the European Resuscitation Council (ERC) guidelines 2010 [12]. Patients receive either manual or mechanical chest compressions (LUCAS ${ }^{\oplus}$ Chest Compression System Physio-Control Inc/Jolife AB, Lund, Sweden or AutoPulse, ZOLL, Chelmsford, MA, USA). Cerebral saturation is continuously measured and registered every four seconds until ALS efforts are terminated in absence of ROSC or until the patient with ROSC arrives at the emergency department (ED). ROSC was defined as return of an organized QRS complex with palpable pulses for more than $20 \mathrm{~min}$. Once ROSC is achieved, the emergency physician presses the event button of the cerebral oximeter and notes the time of ROSC on the Utstein forms $[13,14]$. Targeted temperature management at $33^{\circ} \mathrm{C}$ for $24 \mathrm{~h}$ is initiated at the ED of all participating hospitals. No targeted temperature management was initiated pre-hospital.

Exclusion criteria were age younger than 16 years, technical inability to measure $\mathrm{rSO}_{2}$ (e.g. frontal head trauma), ROSC before or within two minutes after start of $\mathrm{rSO}_{2}$ measurement and known do-notresuscitate status. Patients who already achieved ROSC before $\mathrm{rSO}_{2}$ measurements were initiated, who did not had $\mathrm{rSO}_{2}$ values during the last min before ROSC and patients with incoherence between time notation and $\mathrm{rSO}_{2}$ measurements, were excluded. Patients in whom $5 \%$ or less of the measured overall $\mathrm{rSO}_{2}$ data were registered (the used cerebral saturation device shows an underscore when no $\mathrm{rSO}_{2}$ is being measured), were also excluded from analysis which was then categorized as 'less or no $\mathrm{rSO}_{2}$ values registered'.

Emergency physicians checked $\mathrm{rSO}_{2}$ values to assess the quality of the NIRS signal and as a consequence this study was unblinded. However, $\mathrm{rSO}_{2}$ values were not used in clinical decision making and all patients received ALS according to current ERC guidelines, regardless of $\mathrm{rSO}_{2}$ values [12]. As such, the decision to stop ALS efforts was at the discretion of the medical emergency team and not based on the presented $\mathrm{rSO}_{2}$ values.

Baseline characteristics were prospectively collected from standardized Utstein templates and emergency medical records [13,14]. Digitally recorded time intervals (call to the dispatch center; arrival time of the medical emergency team at the scene) were collected at the dispatch center. The medical emergency team physician noted ALS time intervals such as start of ALS, ROSC and time of discontinuation of ALS. Patients were defined as achieving ROSC if ROSC was sustained for $20 \mathrm{~min}$ or longer. Since the goal of this prospective study was to investigate $\mathrm{rSO}_{2}$ values in OHCA patients during ALS as a prediction for ROSC and to avoid interference from $\mathrm{rSO}_{2}$ measurements when ROSC was already achieved, only data obtained until two minutes before ROSC were used in the data analysis.

\section{Outcome measurements}

Our primary analysis tested the association of rSO2 with ROSC. Change in $\mathrm{rSO}_{2}$ was defined as the absolute change from start of $\mathrm{rSO}_{2}$ measurement until two min before ROSC was clinically determined or until discontinuation of ALS efforts in absence of ROSC. Secondary endpoints were ROSC and favorable neurologic outcome in relation to the first measured $\mathrm{rSO}_{2}$ value, in relation to mean $\mathrm{rSO}_{2}$ during ALS and in relation to a change in $\mathrm{rSO}_{2}$ from one min after start $\mathrm{rSO}_{2}$ measurement until two min before ROSC. According to the GlasgowPittsburgh Cerebral Performance Category (CPC), favorable neurologic outcome 180 days after CA was defined as CPC 1-2 [15-17]. The CPC score has 5 categories: CPC 1 indicates good cerebral performance, CPC 2 moderate cerebral disability (sufficient for independent activities in daily life), CPC 3 implies severe cerebral disability (dependent on others), CPC 4 indicates coma or vegetative state, and CPC 5 stands for death. 


\section{Statistical analysis}

Cerebral oxygen saturation data were downloaded from the portable device according to manufacturer's instructions and exported for statistical analysis. With a sample size of 297 patients (2/1 ratio no ROSC/ROSC), a difference of $6 \%$ in $\mathrm{rSO}_{2}$ increase (sigma 32\%) would be detected with $90 \%$ power, at a two-tailed alpha error rate of $5 \%$. Patients' characteristics were compared using Student's $t$-test if normally distributed and Mann-Whitney test if not normally distributed and expressed as respectively mean \pm standard deviation or median with first and third quartile. Equal distribution was tested by means of the Kolmogorov-Smirnov test. The Chi-square test and Fisher's exact test were used when comparing categorical values. Descriptive statistics were used for continuously measured $\mathrm{rSO}_{2}$ values and are expressed as mean \pm standard deviation or median with first and third quartile, depending on normality. A one-way ANOVA test was used to compare normally distributed continuous data between multiple groups and a Kruskall-Wallis test was performed if the data were not normally distributed. The data as collected are longitudinal in nature: $\mathrm{rSO}_{2}$ was measured repeatedly over time. To take the longitudinal nature of the data into account, a linear mixed model was used. The model allowed for a cubic evolution over time, where the average evolution for the patients who received ROSC and those who did not was allowed to be different. The correlation between the repeated measures of a patient was handled by means of a random intercept and a random slope [18].

Univariate logistic regression was used to investigate the association of every calculated $\mathrm{rSO}_{2}$ variable (initial $\mathrm{rSO}_{2}$, change in $\mathrm{rSO}_{2}$, mean $\mathrm{rSO}_{2}$ and increase in $\mathrm{rSO}_{2}$ of more than 15\%) with ROSC. Hereafter, multivariate logistic regression models were constructed for every $\mathrm{rSO}_{2}$ variable and adjusted for following clinical baseline characteristics; sex, age, witnessed arrest, bystander BLS, bystander defibrillation, initial rhythm, use of mechanical chest compression device, time between emergency call and initiation of ALS and presumed cardiac origin of arrest. Collinearity was tested using variance inflation factor (VIF) where variables with a VIF > 5 were excluded due to multicollinearity. In this manner, the table with values adjusted for sex, age, witnessed arrest, bystander BLS, bystander defibrillation, initial rhythm, use of mechanical chest compression device, time between emergency call and initiation of ALS and presumed cardiac origin of arrest was achieved. A p-value $<0.05$ was considered to be statistically significant. All tests were performed using SPSS 22.00 (SPSS, Chicago, IL, USA). Figures were made using GraphPad Prism 5.01 (GraphPad Software, CA, USA).

\section{Results}

From December 2011 until November 2015, the medical emergency teams of the participating hospitals performed ALS in 746 OHCA patients. In 401 (54\%) patients $\mathrm{rSO}_{2}$ was measured. Of the 401 patients, $329(82 \%)$ patients were enrolled in the study. The reasons why $\mathrm{rSO}_{2}$ was not measured or data were unsuitable for analysis are listed in Fig. 1. In all 329 patients where $\mathrm{rSO}_{2}$ was measured, 180-day outcome data were available. Patient' characteristics and ALS data are presented in Table 1. Patients who achieved ROSC more often had a witnessed CA ( $p<0.001)$, ventricular fibrillation as initial rhythm $(\mathrm{p}<0.001)$, more often underwent early defibrillation by bystanders or paramedics $(\mathrm{p}=.046)$, whereas a mechanical chest compression device was used less often $(\mathrm{p}=.015)$. Patients who achieved ROSC also received less epinephrine $(\mathrm{p}=.001)$. The time between emergency call and start ALS was shorter in the ROSC group ( $\mathrm{p}=.014)$, as well as the duration of resuscitation efforts $(\mathrm{p}<0.001)$, and the time between initiation of ALS and $\mathrm{rSO}_{2}$ measurement $(\mathrm{p}=.028)$.

\section{Primary outcome measurement}

Of the 329 patients, 110 (33\%) achieved ROSC. The course of $\mathrm{rSO}_{2}$ for patients who achieved ROSC and for patients who never achieved ROSC is presented in Fig. 2 (and Fig. S1 in the Supplementary Appendix). The evolution in $\mathrm{rSO}_{2}$ was different between both groups $(\mathrm{p}<0.001)$. The increase in $\mathrm{rSO}_{2}$ measured from start until two minutes before ROSC was higher in the ROSC group (ROSC group median 17\% (IQR 6-29); no-ROSC group median 8\% (IQR 2-13); $\mathrm{p}<0.001)$.

For both patient groups there was an important increase during the first minute (Fig. 2). The median increase in $\mathrm{rSO}_{2}$ after excluding the first minute was still higher in the ROSC group, 9\% (IQR 2-24), than in the no-ROSC group, $2 \%$ (IQR - 2-7) ( $\mathrm{p}<0.001$ ).

If both patients groups, the ROSC and no-ROSC groups, were divided according to magnitude of increase ( $\leq 0 \% ; 0-15 \%$; $15-30 \%$; > 30\%), we observed an absolute increase higher than $15 \%$ more often in patients who achieved ROSC compared to patients who never achieved ROSC (Fig. 3). The unadjusted OR for having an absolute increase of $15 \%$ or more was 4.513 (95\% CI 2.747-7.415; $\mathrm{p}<0.001$ ) in favor of ROSC. Demographic characteristics of both groups, categorized by magnitude of increase are presented in Tables S1 and S2 (in the Supplementary Appendix).

\section{Secondary outcome measurements}

First measured $\mathrm{rSO}_{2}$ was $30 \% \pm 18$ in the ROSC group and $24 \% \pm 15$ in the no-ROSC group $(\mathrm{p}=.004)$.

Higher overall mean $\mathrm{rSO}_{2}$ values until two minutes before ROSC were observed in the ROSC group compared to the no-ROSC group $(41 \% \pm 13$ versus $33 \% \pm 13 ; \mathrm{p}<0.001)$. After adjustment for baseline variables (sex, age, witnessed arrest, bystander BLS, bystander or paramedic defibrillation, initial rhythm, use of mechanical chest compression device, time between emergency call an initiation of ALS and presumed origin of arrest) initial, mean, increase in $\mathrm{rSO}_{2}$ and increase of $\mathrm{rSO}_{2}>15 \%$ remained associated with ROSC (Tables 2 and 3).

Only 19 patients (6\%) had a favorable neurologic outcome (Table S3 in the Supplementary Appendix). Similar analyses as were done for the ROSC versus no-ROSC group were performed in the favorable versus non-favorable neurologic group. Cerebral saturation course from start of $\mathrm{rSO}_{2}$ measurement until two minutes before ROSC or until termination of CPR efforts of both groups is presented in Fig. S2 and S3 (in the Supplementary Appendix). The increase in $\mathrm{rSO}_{2}$ beyond one min after the initiation of $\mathrm{rSO}_{2}$ measurement was higher in the favorable neurologic outcome group compared to the unfavorable neurologic outcome group, $11 \%(3-39)$ versus $4 \%(-1$ to 12$)$ ( $\mathrm{p}=.010)$ respectively. No difference was observed in increase in $\mathrm{rSO}_{2}$ from start measurement between both outcome groups (unfavorable group 9\% (3-17); favorable group $13 \%$ (7-33); $\mathrm{p}=.123$ ). The first measured $\mathrm{rSO}_{2}$ value was higher in the group with favorable neurologic outcome $(35 \% \pm 21)$ compared to the group with an unfavorable neurologic outcome $(26 \% \pm 16)(\mathrm{p}=.016)$. Additionally, the overall mean $\mathrm{rSO}_{2}$ until two minutes before ROSC or until termination of CPR efforts was higher in the favorable neurologic outcome group compared to the one with an unfavorable neurologic outcome, $47 \% \pm 16$ and $35 \% \pm 13$ respectively $(\mathrm{p}=.001)$.

\section{Discussion}

This is the first prospective multicenter study in which $\mathrm{rSO}_{2}$ is measured during pre-hospital ALS in OHCA patients to assess the correlation with ROSC. Cerebral saturation values, measured during prehospital ALS, are associated with ROSC. Increased $\mathrm{rSO}_{2}$ during ALS, definitely more than $15 \%$, appear more predictive for ROSC than the static initial and mean $\mathrm{rSO}_{2}$. The patients who achieved ROSC experienced a higher increase in $\mathrm{rSO}_{2}$ as compared to patients who died prehospital. An absolute increase of $15 \%$ or more was associated with ROSC. Moreover, an increase in $\mathrm{rSO}_{2}$ beyond one min after the 


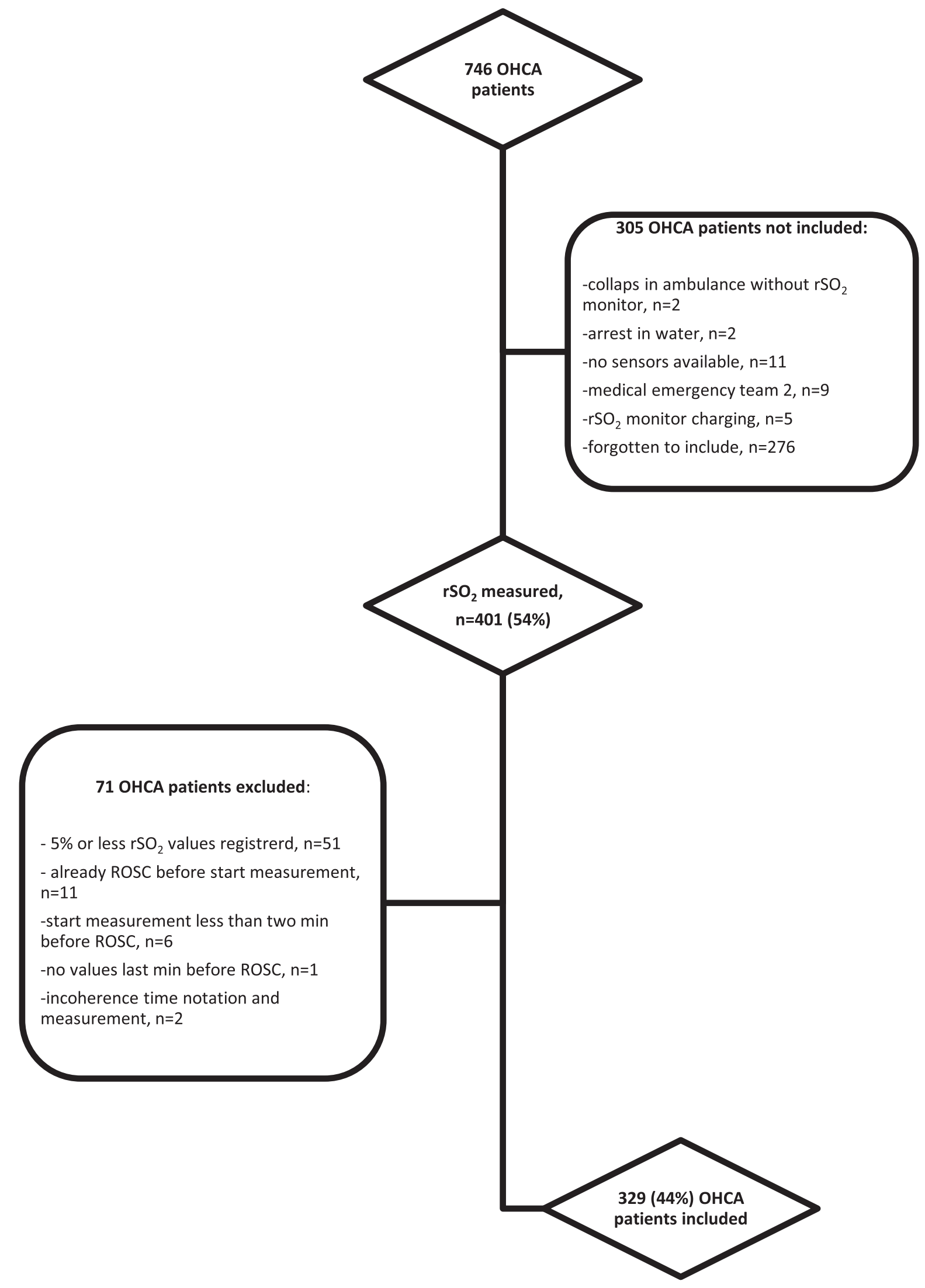

Fig. 1. Flowchart of enrollment and inclusion of study patients.

$\mathrm{N}=$ number of patients; OHCA $=$ out-of hospital cardiac arrest; $\mathrm{ROSC}=$ return of spontaneous circulation; $\mathrm{rSO}_{2}=$ regional cerebral saturation. 
Table 1

Demographic data of study patients.

\begin{tabular}{|c|c|c|c|}
\hline & No-ROSC & ROSC & p-value \\
\hline $\mathrm{N}$ & 219 & 110 & \\
\hline Male & $160(73)$ & $72(65)$ & 0.154 \\
\hline Age & $\begin{array}{l}69 \pm 15 \\
72(58-72)\end{array}$ & $\begin{array}{l}68 \pm 14 \\
70(59-79)\end{array}$ & 0.511 \\
\hline Witnessed arrest & $111(51)$ & $81(74)$ & $<0.001$ \\
\hline BLS $_{\text {bystander }}$ & $86(39)$ & $45(41)$ & 0.774 \\
\hline $\mathrm{BLS}_{\text {paramedic }}$ & $155(71)$ & $80(73)$ & 0.712 \\
\hline Defibrillation $_{\text {bystander/paramedic }}$ & 29 (13) & $24(22)$ & 0.046 \\
\hline \multicolumn{4}{|l|}{ Initial rhythm } \\
\hline Asystole & $140(64)$ & $50(45)$ & 0.001 \\
\hline VF & $31(14)$ & $35(32)$ & $<0.001$ \\
\hline PEA & $48(22)$ & $25(23)$ & 0.868 \\
\hline $\begin{array}{l}\text { Use of mechanical chest compression } \\
\text { device }\end{array}$ & $72(33)$ & $22(20)$ & 0.015 \\
\hline Duration ALS (min) & $\begin{array}{l}29 \pm 18 \\
25(18-35)\end{array}$ & $\begin{array}{l}21 \pm 14 \\
17(12-24)\end{array}$ & $<0.001$ \\
\hline Time $_{\text {EmergencyCall_ALS }}$ (min) & $\begin{array}{l}12 \pm 5 \\
11(8-15)\end{array}$ & $\begin{array}{l}10 \pm 4 \\
10(8-13)\end{array}$ & 0.014 \\
\hline Time $_{\text {StartALS_rSO2measurement }}(\mathrm{min})$ & $\begin{array}{l}6 \pm 7 \\
4(1-9)\end{array}$ & $\begin{array}{l}4 \pm 5 \\
2(0-7)\end{array}$ & 0.028 \\
\hline Dose of adrenaline & $\begin{array}{l}6 \pm 4 \\
5(3-8)\end{array}$ & $\begin{array}{l}5 \pm 3 \\
4(2-6)\end{array}$ & 0.001 \\
\hline Presumed cardiac origin & $153(70)$ & $69(63)$ & 0.192 \\
\hline
\end{tabular}

Data are presented as number (percentage), mean ( \pm standard deviation) and median (IQR).

ROSC $=$ return of spontaneous circulation; BLS = basic life support; $\mathrm{VF}=$ ventricular fibrillation; $\quad \mathrm{PEA}=$ pulseless electrical activity; $\mathrm{ALS}=$ advanced life support; $\mathrm{rSO}_{2}=$ regional cerebral saturation.

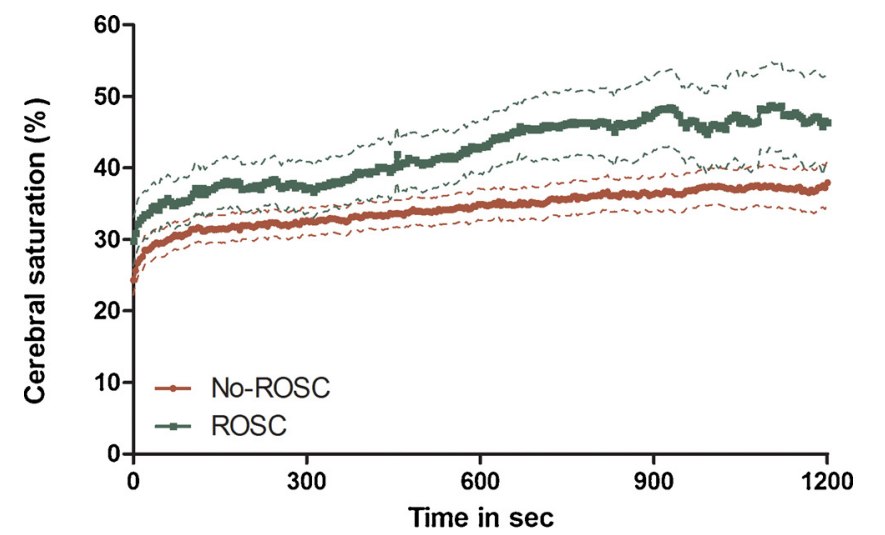

\begin{tabular}{|c|c|c|c|c|}
\hline $\mathrm{N}: 110$ & 101 & 50 & 35 & 25 \\
\hline N: 219 & 211 & 182 & 139 & 97 \\
\hline
\end{tabular}

Fig. 2. Cerebral saturation during advanced life support of patients (mean \pm 95\%CI).

The evolution between both groups was different ( $\mathrm{p}<0.001)$ as also the interaction terms between time and outcome $(\mathrm{p}<0.001)$. ROSC $=$ return of spontaneous circulation; $\mathrm{N}=$ number of patients undergoing ALS.

initiation of $\mathrm{rSO}_{2}$ measurement is associated with long-term favorable neurologic outcome.

In the early pre-hospital phase of an OHCA, the feasibility of $\mathrm{rSO}_{2}$ monitoring had already been demonstrated by others [19-22]. Here, we demonstrated an overall higher mean $\mathrm{rSO}_{2}$ in patients who achieved ROSC ( $41 \%$ versus 33\%) which is in line with previous published $\mathrm{rSO}_{2}$ values measured pre-hospital [20]. Unsurprisingly, the mean $\mathrm{rSO}_{2}$ values are in a lower range compared to mean $\mathrm{rSO}_{2}$ values calculated in IHCA patients [9]. Probably this is due to longer time periods between collapse and initiation of $\mathrm{rSO}_{2}$ measurement. An absolute increase of $15 \%$ or higher in $\mathrm{rSO}_{2}$ was observed more often in patients achieving ROSC. This increase in $\mathrm{rSO}_{2}$ might be a good indicator of response to ALS and ROSC. However, further research is necessary to incorporate

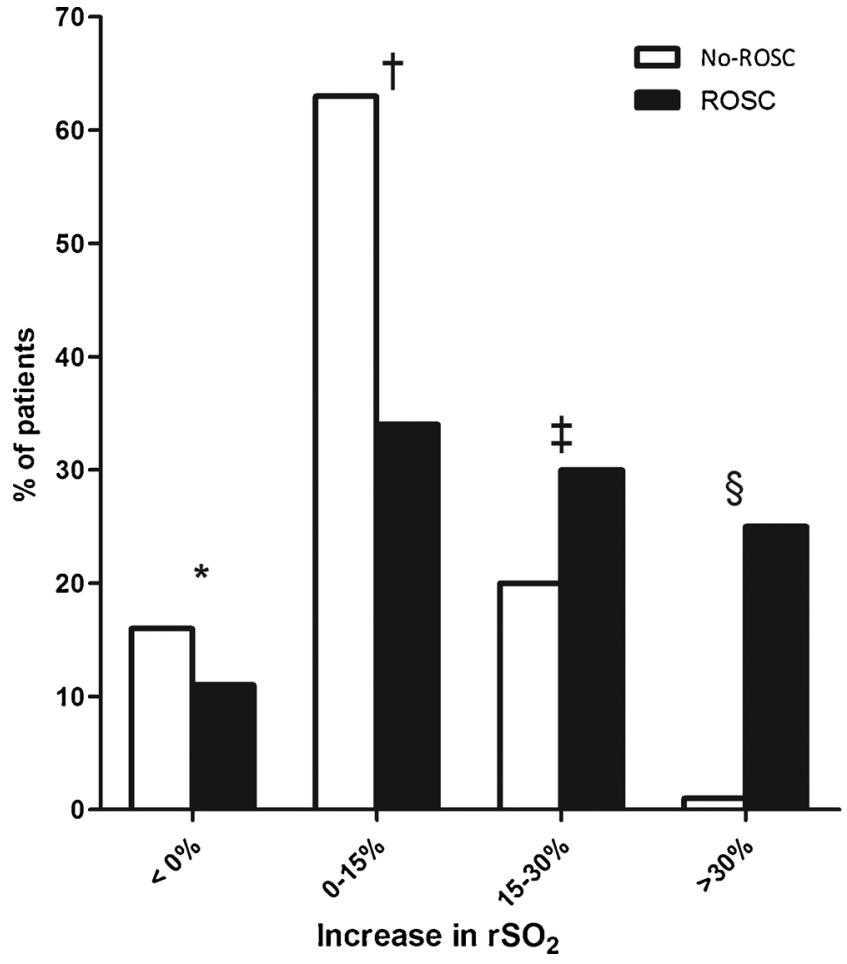

Fig. 3. Increase in cerebral saturation in different categories. $* \mathrm{p}=.255 ; \dagger \mathrm{p}<0.001 ; \nmid \mathrm{p}=.035 ; \S \mathrm{p}<0.001$.

ROSC $=$ return of spontaneous circulation, $\mathrm{rSO}_{2}=$ regional cerebral saturation .

Table 2

Odd ratio of different cerebral saturation variables.

\begin{tabular}{|c|c|c|c|}
\hline & \multicolumn{3}{|c|}{ Unadjusted } \\
\hline & OR & $95 \% \mathrm{CI}$ & p-value \\
\hline Initial $\mathrm{rSO}_{2}$ & 1.020 & $1.005-1.034$ & $<0.001$ \\
\hline Increase $\mathrm{rSO}_{2}$ & 1.059 & $1.039-1.080$ & $<0.001$ \\
\hline Mean $\mathrm{rSO}_{2}$ & 1.047 & $1.027-1.066$ & $<0.001$ \\
\hline$>15 \% \mathrm{rSO}_{2}$ increase & 4.513 & $\begin{array}{r}2.747-7.415 \\
\text { Adjusted }\end{array}$ & $<0.001$ \\
\hline Initial $\mathrm{rSO}_{2}$ & 1.019 & $1.002-1.035$ & 0.026 \\
\hline Increase $\mathrm{rSO}_{2}$ & 1.061 & $1.039-1.084$ & $<0.001$ \\
\hline Mean $\mathrm{rSO}_{2}$ & 1.049 & $1.027-1.071$ & $<0.001$ \\
\hline$>15 \% \mathrm{rSO}_{2}$ increase & 4.878 & $2.788-8.537$ & $<0.001$ \\
\hline
\end{tabular}

Adjusted for sex, age, witnessed arrest, bystander BLS, bystander defibrillation, initial rhythm, use of mechanical chest compression device, time between emergency call and initiation of ALS and presumed cardiac origin of arrest.

this variable in a model with other prehospital variables in order to guide the decision to stop or continue resuscitation more accurately.

Higher initial $\mathrm{rSO}_{2}$ values in the ROSC group could reflect a shorter no-flow time since the time between the emergency call and the start of ALS was shorter in this patient cohort. However, no time $/ \mathrm{rSO}_{2}$ relation curves from onset of arrest until discontinuation of ALS efforts in absence of ROSC or ROSC are currently available. These observations indicate that $\mathrm{rSO}_{2}$ monitoring should be regarded as a trend monitor where a change in $\mathrm{rSO}_{2}$ over time is more informative than a single $\mathrm{rSO}_{2}$ value. Our observed initial $\mathrm{rSO}_{2}$ values were higher than in the study by Storm et al. [21]. Most likely this is caused by the small number of patients included in the latter study.

To date, two multicenter studies used $\mathrm{rSO}_{2}$ monitoring in ALS conditions $[9,23]$. In contrast to our study design, these trials measured $\mathrm{rSO}_{2}$ upon arrival at the ED or in in-hospital cardiac arrest (IHCA) patients. One study aimed to predict neurologic outcome using only the lowest $\mathrm{rSO}_{2}$ value during a one minute $\mathrm{rSO}_{2}$ measurement at arrival of 
Table 3

Increase in $\mathrm{rSO}_{2}$ after adjustment for baseline variables.

\begin{tabular}{lllc}
\hline \multicolumn{3}{l}{ Adjusted } & \\
\cline { 2 - 4 } & OR & $95 \%$ CI & p-value \\
\hline Increase $>15 \%$ & 4.878 & $2.788-8.537$ & $<0.001$ \\
Male & 0.494 & $0.271-0.902$ & 0.022 \\
Age & 0.991 & $0.973-1.010$ & 0.343 \\
Witnessed arrest & 3.029 & $1.674-5.480$ & $<0.001$ \\
BLS $_{\text {bystander }}$ & 0.918 & $0.526-1.602$ & 0.762 \\
Defibrillation & & & \\
Initial rander/paramedic & 1.475 & $0.647-3.363$ & 0.355 \\
Asystole & & & \\
VF & 0.884 & $0.443-1.763$ & 0.658 \\
Use of mechanical chest compression device & 3.243 & $1.319-7.974$ & 0.010 \\
Time & 0.424 & $0.222-0.809$ & 0.009 \\
PresumergencyCall_ALs (min) & 0.963 & $0.907-1.022$ & 0.212 \\
& 0.644 & $0.357-1.163$ & 0.144 \\
\hline
\end{tabular}

$\mathrm{BLS}=$ basic life support; $\mathrm{VF}=$ ventricular fibrillation; PEA = pulseless electrical activity; ALS = advanced life support; $\mathrm{rSO}_{2}=$ regional cerebral saturation.

Baseline variables: sex, age, witnessed arrest, bystander BLS, bystander or paramedic defibrillation, initial rhythm, use of mechanical chest compression device, time between emergency call an initiation of ALS and presumed origin of arrest.

OHCA patients and determined an absolute value of $40 \%$ as $\mathrm{rSO}_{2}$ threshold to predict favorable neurologic outcome [23]. The second multicenter study in IHCA patients showed a correlation between a higher mean $\mathrm{rSO}_{2}$ measured within the last five minutes and ROSC [9]. However, they included the last two minutes before ROSC, possibly overestimating the predictive power of $\mathrm{rSO}_{2}$. They neither measured $\mathrm{rSO}_{2}$ continuously nor explored differences in $\mathrm{rSO}_{2}$ course.

The number of patients with favorable neurologic outcome was low but in line with previously published survival after OHCA [24]. The study was not powered to discriminate for neurologic outcome based on increase in $\mathrm{rSO}_{2}$. This could explain the absence of an observed difference in increase from start $\mathrm{rSO}_{2}$ measurement until two min before ROSC in patients with and without favorable neurologic outcome. A steep initial increase in $\mathrm{rSO}_{2}$ in all patients during the first minute was observed. This increase is most likely related to the initiation of ALS. Therefore it may be clinically relevant to exclude the first minutes of $\mathrm{rSO}_{2}$ measurements and to delay the calculation of increase in $\mathrm{rSO}_{2}$ by one ALS cycle. The evolution of $\mathrm{rSO}_{2}$ one minute after start ALS to two minutes before ROSC or discontinuation ALS efforts may even be an indicator of long-term favorable neurologic outcome.

This study has several limitations. Firstly, we did not measure $\mathrm{rSO}_{2}$ and $\mathrm{ETCO}_{2}$ with the same monitor although the latter is currently the only parameter which can monitor CPR efficacy. Therefore, it was impossible to compare both within an equal time interval. Currently, only one study compared $\mathrm{rSO}_{2}$ and $\mathrm{ETCO}_{2}$ during $\mathrm{CPR}$ in patients admitted to the ED [25]. They found only a poor correlation between both monitoring options with $\mathrm{ETCO}_{2}$ having a higher sensitivity for predicting ROSC while $\mathrm{rSO}_{2}$ having a higher specificity. However, it needs to be stated that $\mathrm{ETCO}_{2}$ data in this study were only noted manually every 1-2 min in contrast to $\mathrm{rSO}_{2}$ values which were saved every four seconds. Secondly, as this is a cohort study, we are not able to conclude that the use of $\mathrm{rSO}_{2}$ will improve the likelihood of achieving ROSC. Thirdly, despite our large number of inclusions, only 19 patients had a favorable neurologic outcome at 180-days post-cardiac arrest, limiting our ability to make strong conclusions on long-term outcome. Only a much larger international multicenter study would be able to answer whether $\mathrm{rSO}_{2}$ measurement can be used in the pre-hospital setting to distinguish patients with a favorable and unfavorable neurologic outcome. Fourthly, as this was a multicenter study with a lot of different medical emergency teams involved, it was not uncommon that not every patient was included. Demanding medical emergency teams to measure $\mathrm{rSO}_{2}$ in an unpredictable and chaotic CA setting is not straightforward. Moreover, it is not always known when the medical emergency team arrives that it will be a cardiac arrest. Therefore, it was to be expected that many eligible patients were not enrolled. Still, we believe that this will be an intrinsic limitation in future studies in the pre-hospital CA setting as well. Finally, emergency physicians were not blinded to the $\mathrm{rSO}_{2}$ measurements, as visual confirmation to assess the quality of the NIRS signal was necessary. Therefore we cannot exclude that $\mathrm{rSO}_{2}$ values unintentionally impacted discontinuation of resuscitation. However, when a correlation between the change in $\mathrm{rSO}_{2}$ and duration of ALS in the no-ROSC group was performed, a negative correlation was observed, with a correlation coefficient of -0.091 and $\mathrm{p}=.177$. This finding suggests no influence of $\mathrm{rSO}_{2}$ values on ALS duration.

In conclusion, we demonstrated the applicability of cerebral saturation monitoring during pre-hospital ALS in a real life multicenter setting. Dynamic cerebral saturation measures during CPR appear to be indicative of ROSC. Certainly, an absolute increase of $15 \%$ or higher in $\mathrm{rSO}_{2}$ during ALS is associated with ROSC.

\section{Conflicts of interest}

None of the authors have any conflicts of interest to declare.

\section{Acknowledgments}

The authors wish to thank the residents, nursing and medical staff of the emergency departments of all participating hospitals for their cooperation and support in this study.

This study is part of the Limburg Clinical Research Program (LCRP) UHasselt-ZOL-Jessa, supported by the foundation Limburg Sterk Merk, Hasselt University, Ziekenhuis Oost-Limburg and Jessa Hospital.

\section{Appendix A. Supplementary data}

Supplementary data associated with this article can be found, in the online version, at https://doi.org/10.1016/j.resuscitation.2018.03.031.

\section{References}

[1] Lemiale V, Dumas F, Mongardon N, et al. Intensive care unit mortality after cardiac arrest: the relative contribution of shock and brain injury in a large cohort. Intensive Care Med 2013(August).

[2] Dragancea I, Rundgren M, Englund E, Friberg H, Cronberg T. The influence of induced hypothermia and delayed prognostication on the mode of death after cardiac arrest. Resuscitation 2013;84(March (3)):337-42.

[3] Soar J, Nolan JP, Böttiger BW, et al. European resuscitation council guidelines for resuscitation 2015: section 3: adult advanced life support. Resuscitation 2015;95(October):100-47.

[4] Murkin JM, Adams SJ, Novick RJ, et al. Monitoring brain oxygen saturation during coronary bypass surgery: a randomized, prospective study. Anesth Analgesia 2007;104(January (1)):51-8.

[5] Denault A, Deschamps A, Murkin JM. A proposed algorithm for the intraoperative use of cerebral near-infrared spectroscopy. Semin Cardiothorac Vasc Anesth 2007;11(December (4)):274-81.

[6] van Bel F, Lemmers P, Naulaers G. Monitoring neonatal regional cerebral oxygen saturation in clinical practice: value and pitfalls. Neonatology 2008;94(4):237-44.

[7] Parnia S, Nasir A, Shah C, et al. A feasibility study evaluating the role of cerebral oximetry in predicting return of spontaneous circulation in cardiac arrest. Resuscitation 2012;83(August (8)):982-5.

[8] Ahn A, Nasir A, Malik H, D'Orazi F, Parnia S. A pilot study examining the role of regional cerebral oxygen saturation monitoring as a marker of return of spontaneous circulation in shockable (VF/VT) and non-shockable (PEA/Asystole) causes of cardiac arrest. Resuscitation 2013(August).

[9] Parnia S, Yang J, Nguyen R, et al. Cerebral oximetry during cardiac arrest: a multicenter study of neurologic outcomes and survival. Crit Care Med 2016(April).

[10] Singer AJ, Ahn A, Inigo-Santiago LA, et al. Cerebral oximetry levels during CPR are associated with return of spontaneous circulation following cardiac arrest: an observational study. Emerg Med J 2014(March).

[11] MacLeod DB, Ikeda K, Vacchiano C, et al. Development and validation of a cerebral oximeter capable of absolute accuracy. J Cardiothorac Vasc Anesth 2012;26(December (6)):1007-14.

[12] Deakin CD, Nolan JP, Soar J, et al. European resuscitation council guidelines for resuscitation 2010 section 4. Adult advanced life support. Resuscitation 2010;81(October (10)):1305-52. 
[13] Jacobs I, Nadkarni V, Bahr J, et al. Cardiac arrest and cardiopulmonary resuscitation outcome reports: update and simplification of the utstein templates for resuscitation registries: a statement for healthcare professionals from a task force of the international liaison committee on resuscitation (American Heart Association, European Resuscitation Council, Australian Resuscitation Council, New Zealand Resuscitation Council, Heart and Stroke Foundation of Canada, InterAmerican Heart Foundation, Resuscitation Councils of Southern Africa). Circulation 2004;110(November (21)):3385-97.

[14] Cummins RO, Chamberlain DA, Abramson NS, et al. Recommended guidelines for uniform reporting of data from out-of-hospital cardiac arrest: the Utstein Style. A statement for health professionals from a task force of the American Heart Association, the European Resuscitation Council, the Heart and Stroke Foundation of Canada, and the Australian Resuscitation Council. Circulation 1991;84(August (2)):960-75

[15] Jennett B, Bond M. Assessment of outcome after severe brain damage. Lancet 1975:1(March (7905)):480-4

[16] Randomized clinical study of thiopental loading in comatose survivors of cardiac arrest. Brain Resuscitation Clinical Trial I Study Group. N Engl J Med 1986;7(February (7)):397-403.

[17] Grenvik A, Safar P, Safar P. Resuscitation after brain ischemia. Brain Failure and Resuscitation. New York, NY: Churchill Livingstone; 1981. p. 155-84.

[18] Verbeke G, Molenberghs G. Linear Mixed Models for Longitudinal Data. 1st ed. New
York: Springer-Verlag; 2000

[19] Meex I, De Deyne C, Dens J, et al. Feasibility of absolute cerebral tissue oxygen saturation during cardiopulmonary resuscitation. Crit Care 2013;2(March (2)):R36.

[20] Schewe J-C, Thudium MO, Kappler J, et al. Monitoring of cerebral oxygen saturation during resuscitation in out-of-hospital cardiac arrest: a feasibility study in a physician staffed emergency medical system. Scand J Trauma Resusc Emerg Med 2014;1(October (1)):58

[21] Storm C, Wutzler A, Trenkmann L, et al. Good neurological outcome despite very low regional cerebral oxygen saturation during resuscitation-a prospective preclinical trial in 29 patients. Scand J Trauma Resusc Emerg Med 2016;24:43.

[22] Genbrugge C, Meex I, Boer W, et al. Increase in cerebral oxygenation during advanced life support in out-of-hospital patients is associated with return of spontaneous circulation. Crit Care 2015;19:112.

[23] Nishiyama K, Ito N, Orita T, et al. Regional cerebral oxygen saturation monitoring for predicting interventional outcomes in patients following out-of-hospital cardiac arrest of presumed cardiac cause: a prospective, observational, multicentre study. Resuscitation 2015(November):135-41.

[24] Gräsner J-T, Lefering R, Koster RW, et al. EuReCa ONE-27 Nations, ONE, Europe, ONE Registry: a prospective one month analysis of out-of-hospital cardiac arrest outcomes in 27 countries in Europe. Resuscitation 2016;105(June):188-95.

[25] Singer AJ, Nguyen RT, Ravishankar ST, et al. Cerebral oximetry versus end tidal CO2 in predicting ROSC after cardiac arrest. Am J Emerg Med 2017(August). 